\title{
A METHOD OF TIME-SERIES CHANGE DETECTION USING FULL POLSAR IMAGES FROM DIFFERENT SENSORS
}

\author{
Wensong Liu ${ }^{1}$, Jie Yang ${ }^{1 *}$, Jinqi Zhao ${ }^{1}$, Hongtao Shi ${ }^{1}$, Le Yang ${ }^{1}$
}

\begin{abstract}
${ }^{1}$ The State Key Laboratory of Information Engineering in Surveying, Mapping and Remote Sensing, Wuhan University, 430079 Wuhan, China- liuwensongupc@163.com; yangj@whu.edu.cn; masurq@whu.edu.cn; shihongtaosg@163.com; yangleupc@163.com
\end{abstract}

\section{ICWG III/IVb}

KEY WORDS: PolSAR; time-series change detection; omnibus statistic test; generalized Gaussian mixture model (GGMM); different sensors

\begin{abstract}
:
Most of the existing change detection methods using full polarimetric synthetic aperture radar (PolSAR) are limited to detecting change between two points in time. In this paper, a novel method was proposed to detect the change based on time-series data from different sensors. Firstly, the overall difference image of a time-series PolSAR was calculated by ominous statistic test. Secondly, difference images between any two images in different times ware acquired by $R_{j}$ statistic test. Generalized Gaussian mixture model (GGMM) was used to obtain time-series change detection maps in the last step for the proposed method. To verify the effectiveness of the proposed method, we carried out the experiment of change detection by using the time-series PolSAR images acquired by Radarsat-2 and Gaofen-3 over the city of Wuhan, in China. Results show that the proposed method can detect the time-series change from different sensors.
\end{abstract}

\section{INTRODUCTION}

Due to the all-time and all-weather mapping capability of synthetic aperture radar (SAR), which is independent of, for instance, cloud cover, night-time, and severe weather, change detection of SAR has played an important role in the understanding of the relationships and interactions between people and natural phenomena in recent years (Bruzzone, 2013; Hachicha, 2014; Singh, 1989). Change detection with SAR images has been used in applications such as disaster monitoring (Conradsen, 2003; Giustarini, 2013), ecological monitoring (Zhao, 2014; Zhao, 2016), regulatory policy development (Sun, 2016; Zhao, 2013), and environmental impact assessment (Zhao, 2014). The Chinese Gaofen-3 (GF-3) satellite was launched on 10 August, 2016.It is the first multipolarization SAR imaging satellite to be developed in China and it has promoted research into change detection based on SAR images(Sun, 2017).Meanwhile, full polarimetric synthetic aperture radar (PolSAR) data contain both phase and amplitude information from radar returns transmitted in two different polarizations, signifying that more scattering information can be used for change detection(Zhao, 2017). Therefore, many researchers have focused on change detection using bi-temporal PolSAR images (Liu, 2014; Moser, 2006; Sumaiya, 2016).

Although the traditional pixel-based unsupervised change detection method using bi-temporal PolSAR images can detect the change between two different times with same sensor (Akbari, 2016), they could not detect the total changes of the entire time series from different sensors (Conradsen, 2016). To solve these problems, some researchers simply compared the pair-wise images in the time-series images and detected the time-series change, which suffers from some deficiencies, such as it is time-consuming and couldn't detect some small continuous changes (Conradsen, 2016). When analyze the difference images, traditional methods such as two-dimensional entropic segmentation (TDES) algorithm (Jansing, 1999), Otsu's algorithm (Otsu, 1975), improved K\&I algorithm (Zhao, 2017) and Kapur entropy algorithm (Yetgin, 2012) and so on, assume the probability density function (PDF) of the difference image complies with the Gaussian distribution. While the difference images calculated by ominous statistic test and $R_{j}$ statistic test algorithm are not complies with the Gaussian distribution (Conradsen, 2016). In consequence, traditional algorithm is not suitable for the analysis of difference image which obtained by ominous or $\mathrm{R}_{\mathrm{j}}$ statistic test algorithm. Fortunately, Gaussian mixture models (GMM) can fit any distribution of data (Rasmussen, 2000). In our previous work (Liu, 2017), by improving the GMM algorithm, it can better adapt to the analysis of difference image.

Focusing on above problems, a method (named OS_GMM) of time series change detection using full PolSAR images from different sensors is proposed in this paper. OS_GMM integrates the respective advantages of the omnibus test (Conradsen, 2016) and generalized GMM (Liu, 2018) techniques. The proposed method should not be regarded as a combination of many methods but as a fully unified algorithm with different steps. In this method, the use of the omnibus statistic is an effective strategy for obtaining the difference images from PolSAR timeseries images, and we use the generalized GMM algorithm to help us to choose the threshold of change detection map.

\section{METHODOLOGY}

\subsection{Omnibus Test}

The omnibus statistic test algorithm effectively utilizes polarimetric and temporal information from time-series PolSAR images. The PolSAR image includes the backscattering

\footnotetext{
*Correspondence: yangj@whu.edu.cn
} 
coefficients of the four polarimetric channels of the object (Conradsen, 2003). For orthogonal polarizations basis, the scattering information of ground objects can be represented by the following covariance matrix C:

$$
C=\left\langle\left[\begin{array}{lll}
\left|S_{h h}\right|^{2} & S_{h h} S_{h v}^{*} & S_{h h} S_{v v}^{*} \\
S_{h v} S_{h h}^{*} & \left|S_{h v}\right|^{2} & S_{h v} S_{v v}^{*} \\
S_{v v} S_{h h}^{*} & S_{v v} S_{h v}^{*} & \left|S_{v v}\right|^{2}
\end{array}\right]\right\rangle
$$

Different elements in covariance matrix $\mathrm{C}$ represent the backscattering coefficients. For the multi-look conditions, the covariance matrix $\mathrm{C}$ of PolSAR image obeys the complex Wishart distribution $\left(X \in W\left(p, n, \Sigma_{X}\right)\right)$ and probability density functions (PDF) of $\mathrm{C}$ can be described as follows.

$$
\begin{aligned}
& f(C)=\frac{1}{\Gamma_{n}(p)} \frac{1}{\left|\Sigma_{C}\right|^{n}}|C|^{n-p} \exp \left\{-\operatorname{tr}\left[\Sigma_{C}^{-1} C\right]\right\} \\
& \Gamma_{p}(n)=\pi^{p(p-1) / 2} \prod_{j=1}^{p} \Gamma(n-j+1)
\end{aligned}
$$

Where, $\operatorname{tr}(\cdot)$ is the trace of covariance matrix $\mathrm{C}, \mathrm{n}$ is the number of look of PolSAR image, and $p$ represents the dimension of matrix C. For fully PolSAR image, $p$ is equals to 3 (Conradsen, 2016).

Assuming that the multi-parameters $\sum_{X_{1}}, \Sigma_{X_{2}}, \ldots \Sigma_{X_{j-1}}, \Sigma_{X_{j}}, \ldots \Sigma_{X_{k}}$ of time-series $\left(t_{1}<t_{2}<\ldots<t_{k}\right)$ PolSAR images are independent, and they obey the complex Wishart distribution:

$$
\begin{aligned}
X_{1} & \in W\left(p, n_{1}, \Sigma_{X_{1}}\right) \\
X_{2} & \in W\left(p, n_{2}, \Sigma_{X_{2}}\right) \\
& \ldots \\
X_{k} & \in W\left(p, n_{k}, \Sigma_{X_{k}}\right)
\end{aligned}
$$

where $\mathrm{p}$ represents the dimension of $X_{1}, X_{2}, \ldots X_{k}$, $n_{1}, n_{2}, \ldots n_{k}$ is the number of look of $X_{1}, X_{2}, \ldots X_{k}$, and $\Sigma_{X_{1}}, \Sigma_{X_{2}}, \ldots \Sigma_{X_{k}}$ represent the scattering matrix of $X_{1}, X_{2}, \ldots X_{k}$.

According to omnibus statistic test theory, $H_{0}$ hypothesis can be described as $H_{0}: \sum_{X_{1}}=\sum_{X_{2}}=\ldots=\sum_{X_{j-1}}=\sum_{X_{j}}=\ldots \sum_{X_{k}}$, which means the matrixes of time-series PolSAR images are equal. In other words, if $H_{0}$ hypothesis were the case, the feature has not changed in the time interval $\left[\boldsymbol{t}_{1}, \boldsymbol{t}_{\boldsymbol{k}}\right]$. On the contrary, the feature has at least one change in the time-series $\left[\boldsymbol{t}_{1}, \boldsymbol{t}_{k}\right]$ of PolSAR images.

Supposing that the omnibus statistic test based on maximum likelihood estimation (MLE) has a joint density $f\left(\sum_{X_{1}}, \Sigma_{X_{2}} \ldots \Sigma_{X_{k}}, \theta\right)$, where $\theta$ is the set of parameters of the probability function that has generated the data. $H_{0}$ states that $\theta \in H_{0}$, and the likelihood ratio of the omnibus statistic test is shown as follows:

$$
Q=\frac{\max _{\theta \in H_{0}} L(\theta)}{\max _{\theta \in \Omega} L(\theta)}=\frac{L\left(\sum_{S}\right)}{\prod_{i=1}^{i=k} L_{X_{1}}\left(\Sigma_{X_{i}}\right)}
$$

where,

$$
\begin{aligned}
& \prod_{i=1}^{i=k} L_{X_{i}}\left(\Sigma_{X_{i}}\right)=\frac{1}{\prod_{i=1}^{i=k} \Gamma_{p}\left(n_{i}\right)} \prod_{i=1}^{i=k}\left|\Sigma_{X_{i}}\right|^{-n_{i}} \prod_{i=1}^{i=k}\left|\Sigma_{X_{i}}\right|^{n_{i}-p} \exp \left\{-\operatorname{tr}\left(\sum_{i=1}^{i=k} \Sigma_{X_{i}}^{-1} X_{i}\right)\right\} \\
& L\left(\Sigma_{S}\right)=\frac{1}{\prod_{i=1}^{i=k} \Gamma_{p}\left(n_{i}\right)}|\Sigma|^{-\sum_{i=1}^{i=k} n_{k}} \prod_{i=1}^{i=k}\left|\Sigma_{X_{i}}\right|^{n_{i}-p} \exp \left\{-\operatorname{tr}\left(\Sigma^{-1}|X|\right)\right\}
\end{aligned}
$$

If $n_{1}=n_{2}=\cdots=n_{k}=n$, this leads to the desired likelihood-ratio omnibus statistic test:

$$
Q=\frac{\max _{\theta \in H_{0}} L(\theta)}{\max _{\theta \in \Omega} L(\theta)}=\frac{L\left(\sum_{S}\right)}{\prod_{i=1}^{i=k} L_{X_{1}}\left(\Sigma_{X_{i}}\right)}=\left\{k^{p k} \frac{\prod_{i=1}^{i=k}\left|X_{i}\right|}{|X|^{k}}\right\}^{n}
$$

where $X=\sum_{i=1}^{i=k} X_{i}, X_{i}=n\langle C\rangle_{i}$ and Equation (6) in logarithm form as follows:

$$
\ln Q=n\left\{p k \ln k+\sum_{i=1}^{i=k} \ln \left|X_{i}\right|-k \ln |X|\right\}
$$

In general, the overall similarity of time-series PolSAR images is measured by $-\ln Q$. The larger the value, the greater the probability that change will generally occur in time-series PolSAR images.

\section{$2.2 \mathbf{R}_{\mathbf{j}}$ Statistic Test}

The omnibus statistic test algorithm can be used to detect the overall change of the time-series PolSAR images, but it is limited to detecting the change between two any different times. To compensate for the shortcoming of omnibus statistic test, Conradsen et al. (Conradsen, 2016) formulated the $R_{j}$ statistic test algorithm, which is used to generate the different images between any two images in different times (Conradsen, 2016).

According to $R_{j}$ statistic test, if the matrixes of any two different PolSAR images in time are equal $\left(H_{0}: \sum_{X_{j-1}}=\Sigma_{X_{j}}\right)$, it indicates that there is no change in the time interval [ $\left.\boldsymbol{t}_{\boldsymbol{j}-\mathbf{1}}, \boldsymbol{t}_{\boldsymbol{j}}\right]$. Instead, if the matrixes are not equal $\left(H_{1}: \sum_{X_{j-1}} \neq \sum_{X_{j}}\right.$ ), the change happens between the two images. According to $R_{j}$ statistic test, the likelihood ratio of the statistic can be shown as follows: 


$$
\begin{aligned}
R_{j} & =\frac{j^{j p n}}{(j-1)^{(j-1) p n}} \frac{\left|X_{1}+\ldots+X_{j-1}\right|^{(j-1) n}\left|X_{j}\right|^{n}}{\left|X_{1}+\ldots+X_{j}\right|^{j n}} \\
& =\left\{\frac{j^{j p}}{(j-1)^{(j-1) p}} \frac{\left|X_{1}+\ldots X_{j-1}\right|^{(j-1)}}{\left|X_{1}+\ldots X_{j}\right|^{j}}\right\}^{n}
\end{aligned}
$$

Equation (8) in logarithm form is as follows:

$$
\begin{aligned}
\ln R_{j} & =n\{p(j \ln j-(j-1) \ln (j-1)) \\
& +(j-1) \ln \left|\sum_{i=1}^{j-1} X_{i}\right|+\ln \left|X_{j}\right|-j \ln \left|\sum_{i=1}^{j} X_{i}\right|
\end{aligned}
$$

Similarly, the similarity of PolSAR images from two any different times is measured by $-\ln R_{j}$. The larger the value, the greater the probability that change will occur between the two images.

\subsection{The Generalized Gaussian Mixture Model (GGMM) and Expectation Maximization (EM)}

The GMM (Rasmussen, 2000) is an unsupervised statistical learning approach that is widely used in statistical pattern recognition. The GMM can represent an arbitrarily complex PDF and is suitable to fit an unknown data distribution of the difference images. The finite GMM with k components can be calculated as:

$$
\begin{gathered}
f(x)=\sum_{i=1}^{k} \alpha_{i} p\left(x \mid \theta_{i}\right) \\
\theta=\left\{\mu_{1}, \ldots, \mu_{m}, \sigma_{1}^{2}, \ldots, \sigma_{m}^{2}\right\}
\end{gathered}
$$

where, $\alpha_{m}$ represent the mixing probabilities of the mth component, which must be positive and sum to one; $\mu_{k}$ are the means; $\sigma_{k}^{2}$ are the variances; and $p\left(x \mid \theta_{m}\right)$ is a normalized Gaussian with specified mean and variance.

In general, the distribution of difference image $\left(p\left(x_{d}\right)\right)$ can be modeled as a weighted sum of two distributions. One represents the 'changed' class and another represents 'unchanged' class data distributions (Celik, 2011), i.e.

$$
p\left(x_{\mathrm{d}}\right)=p\left(\mathrm{w}_{\mathrm{u}}\right) p\left(x_{\mathrm{d}} \mid w_{u}\right)+p\left(\mathrm{w}_{\mathrm{c}}\right) p\left(x_{\mathrm{d}} \mid w_{c}\right)
$$

where $p\left(x_{\mathrm{d}} \mid \mathrm{w}_{\mathrm{u}}\right)$ and $p\left(x_{\mathrm{d}} \mid \mathrm{w}_{c}\right)$ are posteriori PDFs, and $p\left(w_{c}\right)$ and $p\left(w_{u}\right)$ are priori probabilities of classes $w_{u}$ and $w_{c}$, respectively. The data distribution $p\left(x_{d}\right)$ can also be modeled using the k-component GMM (Zivkovic, 2004), i.e.

$$
p\left(x_{d}\right)=\sum_{k=1}^{K} p(k) p\left(x_{d} \mid k\right)=\sum_{\forall \theta_{u} \in M_{u}} p\left(\theta_{u}\right) p\left(x \mid \theta_{u}\right)+\sum_{\forall \theta_{c} \in M_{c}} p\left(\theta_{c}\right) p\left(x \mid \theta_{c}\right)
$$

where $p(k)$ is the prior probability of the data point having been generated from component $\mathrm{n}$ of the mixture, and $p\left(\mathrm{x}_{\mathrm{d}} \mid k\right)$ is the nth component density modeled with a Gaussian density function. The data distribution modeled with Equation (11) can be separated into two distributions representing the data distributions of the 'changed' and 'unchanged' classes.

In order to estimate $\theta$, it is usual to introduce the $\log$ likelihood function defined as shown in Equation (13):

$\log p(x \mid \theta)=\log \prod_{i=1}^{n} p\left(x^{(\mathrm{i})} \mid \theta\right)=\sum_{i=1}^{n} \log \sum_{m=1}^{k} \alpha_{m} p\left(x^{(\mathrm{i})} \mid \theta_{m}\right)$

The EM algorithm is an iterative method used to find the maximum likelihood or the maximum a posteriori estimates of the components in the GMM (Dempster, 1977). The EM iteration alternates between performing an expectation (E) step and a maximization (M) step. The E-step creates a function for the expectation of the log-likelihood evaluated using the current estimate for the parameters:

$$
Q(\theta, \hat{\theta}(\mathrm{t})) \equiv \mathrm{E}[\log p(X, \gamma \mid \theta) \mid X, \hat{\theta}(\mathrm{t})]=\log p(X, \hat{\gamma} \mid \theta)
$$

where

$$
\hat{\gamma}_{i m} \equiv E\left[\gamma_{i m} \mid x, \hat{\theta}(\mathrm{t})\right]=\frac{\hat{\alpha}_{m}(\mathrm{t}) p\left(x_{i} \mid \hat{\theta}_{m}(\mathrm{t})\right)}{\sum_{j=1}^{k} \hat{\alpha}_{j}(\mathrm{t}) p\left(x_{i} \mid \hat{\theta}_{j}(\mathrm{t})\right)}
$$

The M-step computes the parameters maximizing the expected log-likelihood found in the E-step.

$$
\frac{\partial(Q(\theta, \hat{\theta}(\mathrm{t})))}{\partial \theta}=0
$$

We update the parameter estimation according to Equation (16):

$$
\begin{gathered}
\alpha_{i}=\sum_{i=1}^{n} \hat{\gamma}_{i m} / n \\
\mu_{i}=\sum_{i=1}^{n} \hat{\gamma}_{i m} x_{i} / \sum_{i=1}^{n} \hat{\gamma}_{i m} \\
\sigma_{i}^{2}=\sum_{i=1}^{n} \hat{\gamma}_{i m}\left(x_{i}-\mu_{i}\right)^{T}\left(x_{i}-\mu_{i}\right) / d \sum_{i=1}^{n} \hat{\gamma}_{i m}
\end{gathered}
$$

The EM algorithm of the criterion of convergence is defined as:

$$
\|\theta-\hat{\theta}(\mathrm{t})\|<\delta
$$

For a certain class of traditional GMM algorithm, there is an important parameter commonly referred to as $\mathrm{K}$ that specifies the number of components to detect (Liu, 2017). However, the correct choice of $\mathrm{K}$ is often ambiguous. Fortunately, it can avoid the use of empirical methods for the selection of the number of components for the GMM and find the optimal number of components $\mathrm{K}$ in our previous work (Liu, 2017).

\subsection{The Proposed Method of Time-Series Change Detection Using Images from Different Sensors}

The procedure of time-series unsupervised change detection based on different sensors is shown as Figure 1. 


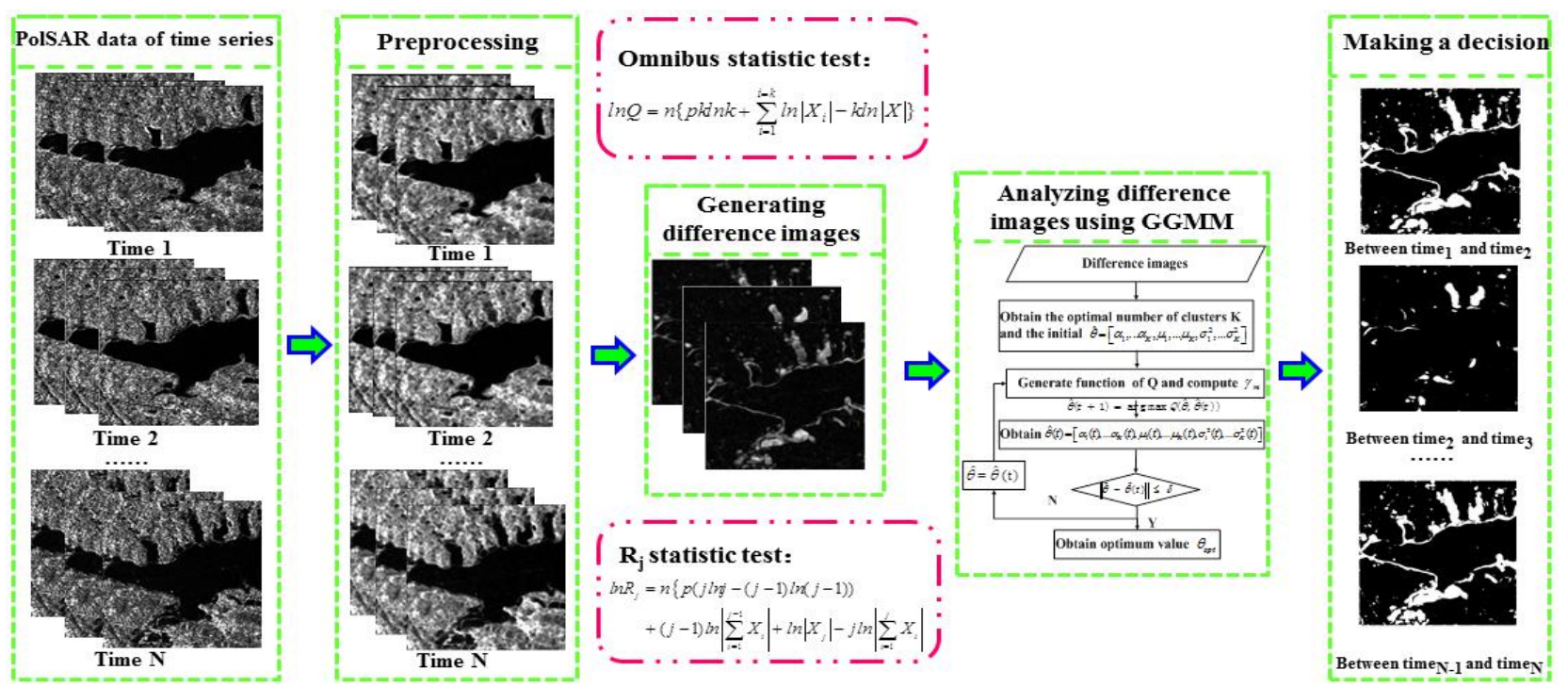

Figure 1. The process flow of the proposed method.

The process of change detection includes, 1) Data preprocessing, including radiation correction, geometric correction, coregistration and filtering. 2) Calculating the overall difference image of a time-series image by ominous statistic test and acquiring the difference images between any two images in different times by $R_{j}$ statistic test. 3) Modeling the difference images by GGMM model, obtaining the statistic distribution of changeable and non-changeable classes. 4) Making decision analysis according to formula (19) and calculating the change.

$C D(\mathrm{i}, \mathrm{j})=\left\{\begin{array}{l}255, p\left(w_{u}\right) p\left(x \mid w_{u}\right)<p\left(w_{c}\right) p\left(x \mid w_{c}\right) \\ 0, \text { otherwise }\end{array}\right.$

where, ' 0 ' represents non-changeable class and ' 255 ' represents changeable classes.

\section{EXPERIMENTS AND RESULTS}

\subsection{Study Area and Background}

Wuhan (as shown in Figure 2) lies East Longitude $113^{\circ} 41^{\prime}$ $115^{\circ} 05^{\prime}$, North Latitude $29^{\circ} 58^{\prime}-31^{\circ} 22^{\prime}$, and it is the only megalopolises in the China West. Wuhan is known as 'River City' because the third largest river (Yangtze River) in the world and the largest tributary of the Yangtze River (Han River) across this city and many lakes were formed, such as East Lake, LiangZi Lake and so on. Dramatic changes have taken place in the city of Wuhan during the 'Twelfth Five-Year Plan' period (from 2011 to 2015). In order to detect the dramatic changes of city, time-series PolSAR images were acquired by Radarsat-2 and GF-3 sensors. In this study, our aim was to detect the dramatic changes of city and monitor the changes associated with the tunnel construction on East Lake.

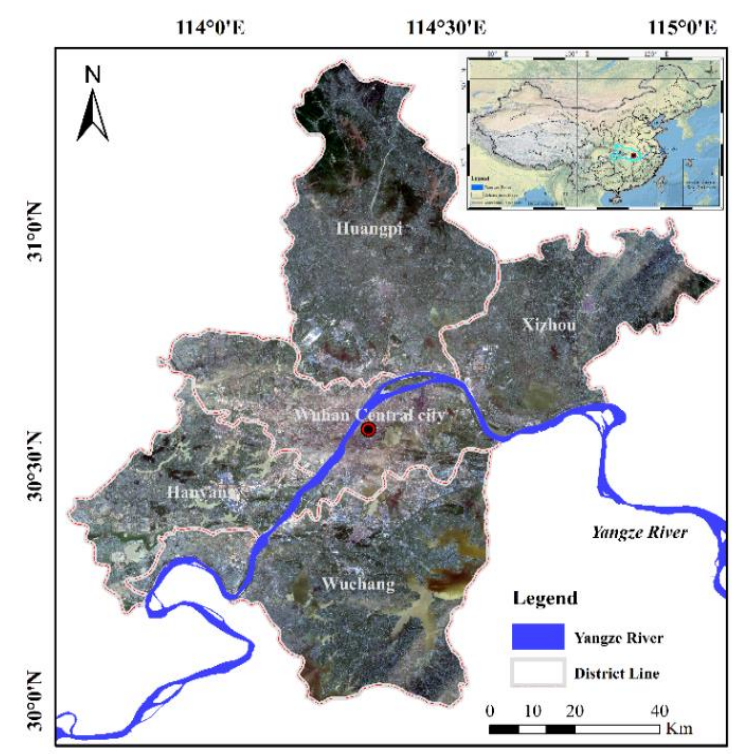

Figure 2. Location of the study area.

The preprocessing of PolSAR time-series images is important for change detection. In this study, the preprocessing consisted of radiometric calibration, speckle filtering, geometric correction and image co-registration. After the radiometric calibration, the pixel values of the PolSAR time-series images from different sensors are directly related to the radar backscatter of the scene. This condition is necessary for the comparison of PolSAR images acquired at different dates. Speckle filtering and image co-registration were also performed. From 2011 to 2017, construction of a new tunnel and its ancillary buildings took place on East Lake in Wuhan, China. After the new tunnel constructed, the ancillary buildings were removed in 2016. The land-cover types of this area are lake, bridge, city, and forest. PolSAR images acquired by Radarsat- 2 and GF-3 sensors display the changes associated with the construction of the new tunnel on East Lake and the RGB 
images(1200 rows, 1000 columns) in Pauli basis $\left(\left|S_{h h}-S_{v v}\right|\right.$ for red, $\left|S_{h v}\right|$ for green, and $\left|S_{h h}+S_{v v}\right|$ for blue) are shown in Figure 3.

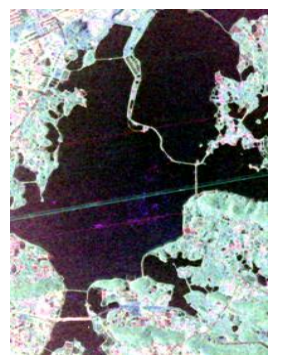

(a)

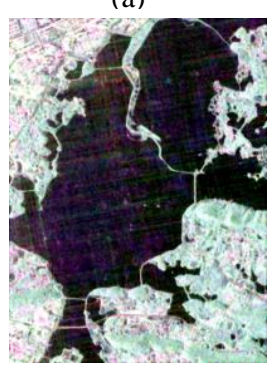

(d)

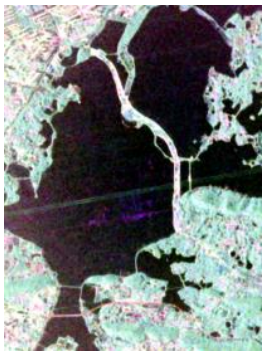

(b)

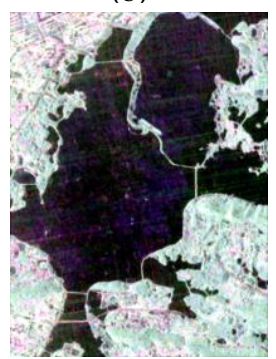

(e)
Figure 3. RADARSAT-2 PolSAR images acquired on (a) December 07, 2011. (b) June 25, 2015. (c) July 6, 2016; and GF-3 PolSAR images acquired on (d) April, 30, 2017. (e) May, 29, 2017.

The validity of the proposed algorithm applied to different sensors is verified in this part. OS_GMM method was used to detect the change in the construction process of East Lake Tunnel. Dramatic changes have taken place around East Lake from 2011 to 2017 and the changes of different period over East Lake are shown in Figure 4.

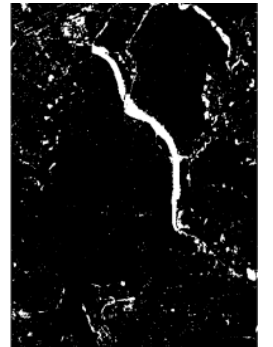

(a)

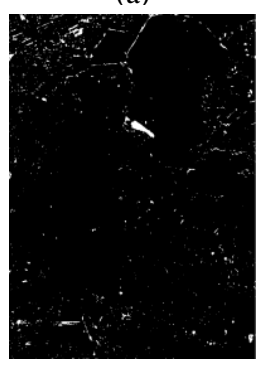

(d)

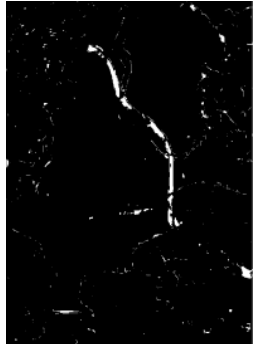

(b)

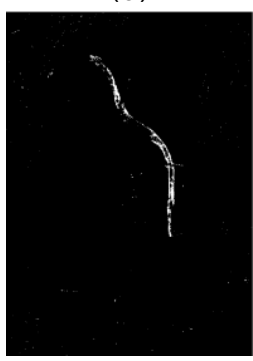

(e)

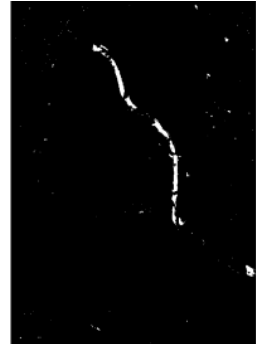

(c)

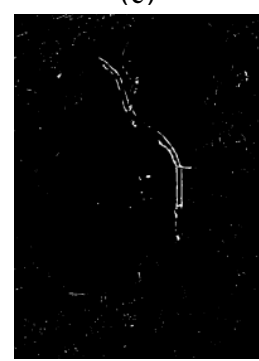

(f)

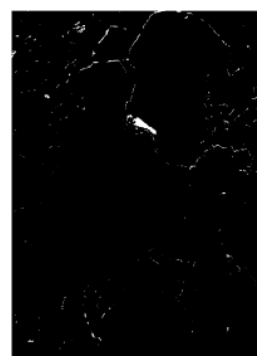

( g)

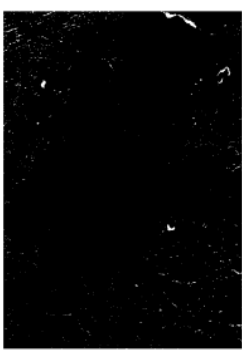

(j)

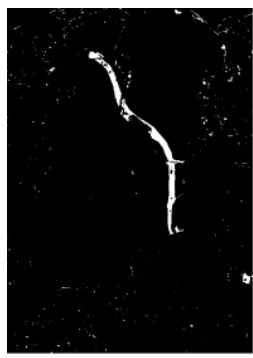

(h)

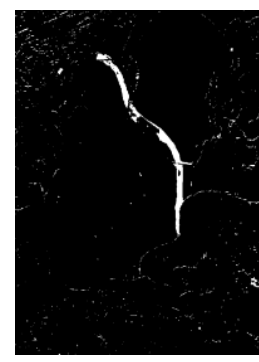

(i)
Figure 4. Time-series change detection over tunnel of East Lake from 2011 to 2017(a) between December, 2011 and June, 2015.(b) between December, 2011 and July, 2016.(c) between June, 2015 and July, 2016.(d) between December, 2011 and April, 2017.(e) between June, 2015 and April, 2017.(f) between July, 2016 and April, 2017.(g) between December, 2011 and May, 2017.(h) between June ,2015 and May, 2017.(i) between July ,2016 and May, 2017.(j)between April, 2017and May, 2017.

To give a visual impression of the results, Figure 4 shows the time-series change detection results over the East Lake tunnel from 2011 to 2017. Figure 4a shows the change detection result from December 2011 to June 2015, where the changes reflect the construction of the new tunnel and urbanization. Figure $4 \mathrm{~b}$ shows the change detection result from 2011 to 2016, where the changes again reflect the construction of the new tunnel and urbanization. Figure $4 \mathrm{c}$ shows the change detection result from June 2015 to July 2016, where the changes reflect the removal of the ancillary buildings and urbanization. Figures $4 \mathrm{~d}$ and $4 \mathrm{~g}$ show the change detection result from December 2011 to April 2017 and from December 2011 to May 2017, where the changes reflect the urbanization. Figures $4 \mathrm{e}$ and $4 \mathrm{~h}$ show the change detection result from December 2011 to April 2017 and from June 2015 to May 2017, respectively, where the changes reflect the removal of the ancillary buildings and urbanization. Figures 4f and 4i show the change detection result from July 2016 to April 2017 and from July 2016 to May 2017, where the changes reflect the removal of ancillary buildings. Figure $4 \mathrm{j}$ shows the change detection result from April 2017 to May 2017, where few changes can be observed.

\section{CONCLUSIONS}

Most of existing unsupervised change detection methods using PolSAR images are limited to detecting change between two points in time, in this paper, omnibus statistic test algorithm is designed to detect the change over the entire time period, and $R_{j}$ 
statistic test is used to detect changes in different time interval. The proposed approach is based on automatic generation of the different images using an omnibus statistic and a statistical clustering approach. The omnibus statistic is a good strategy for obtaining the difference images from time series PolSAR images from different sensors. Using the proposed method, we were able to accurately detect the changes associated with the construction of a tunnel on East Lake from 2011 to 2017, dramatic changes in the city of Wuhan during 'Twelfth FiveYear Plan period'. The experimental results indicates that the proposed method can detect changes in a series of images from Radarsat-2 and GF-3 sensors. However, some further improvements are still necessary in future. For example, the results of change detection are easy affected by the speckle noise when use PolSAR images, and the method may be timeconsuming to arrive at the convergence criterion in this paper.

\section{ACKNOWLEDGEMENTS}

The authors would like to thanks the National Natural Science Foundation of China, No. 91438203, No. 61371199, No. 41501382, No.41601355; Public Welfare Project of Surveying and Mapping Interest (201412002); Hubei Provincial Natural Science Foundation (No. 2015CFB328, No.2016CFB246); National Basic Technology Program of Surveying and Mapping (No. 2016KJ0103); Technology of target recognition based on GF-3(No. 03-Y20A10-9001-15/16).

\section{REFERENCES}

Akbari, V.; Anfinsen, S.N.; Doulgeris, A.P.; Eltoft, T.; Moser, G.; Serpico, S.B. Polarimetric SAR Change Detection With the Complex Hotelling-Lawley Trace Statistic. IEEE Trans. Geosci. Remote Sens. 2016, 54, 3953-3966.

Bouyahia, Z.; Benyoussef, L.; Derrode, S. Change detection in synthetic aperture Radar images with a sliding hidden Markov chain model. J. Appl. Remote Sens. 2008, 2, 513-526.

Bruzzone, L.; Prieto, D.F. A minimum-cost thresholding technique for unsupervised change detection. Int. J. Remote Sens. 2000, 21, 3539-3544.

Bruzzone, L.; Bovolo, F. A novel framework for the design of change-detection systems for very-high-resolution remote sensing images. Proc. IEEE. 2013, 101, 609-630.

Bunch, J.R.; Fierro, R.D. A constant-false-alarm-rate algorithm. Linear Algebr Appl. 1992, 172, 231-241.

Carincotte, C.; Derrode, S.; Bourennane, S. Unsupervised change detection on SAR images using fuzzy hidden Markov chains. IEEE Trans. Geosci. Remote Sens. 2006, 44, 432-441.

Celik, T.; Ma, K.-K. Multitemporal Image Change Detection Using Undecimated Discrete Wavelet Transform and Active Contours. IEEE Trans. Geosci. Remote Sens. 2011, 49, 706716 .

Conradsen, K.; Nielsen, A.A.; Schou, J.; Skriver, H. A test statistic in the complex wishart distribution and its application to change detection in polarimetric SAR data. IEEE Trans. Geosci. Remote Sens. 2003, 41, 4-19.
Conradsen K, Nielsen A A, Skriver H. Determining the points of change in time series of polarimetric SAR data. IEEE Transactions on Geoscience and Remote Sensing, 2016, 54(5): 3007-3024

Dempster, A.P.; Laird, N.M.; Rubin, D.B. Maximum likelihood from incomplete data via the EM algorithm. J. R. Stat. Soc. Ser. B (Methodol.) 1977, 39, 1-38.

Giustarini, L.; Hostache, R.; Matgen, P.; Schumann, G.J.-P.; Bates, P.D.; Mason, D.C. A change detection approach to flood mapping in urban areas using TerraSAR-X. IEEE Trans. Geosci. Remote Sens. 2013, 51, 2417-2430.

Goutte, C.; Toft, P.; Rostrup, E.; Nielsen, F.; Hansen, L.K. On clustering fMRI time series. Neuroimage 1999, 9, 298-310.

Hachicha, S.; Chaabane, F. On the SAR change detection review and optimal decision. Int. J. Remote Sens. 2014, 35, 1693-1714.

Inglada, J.; Mercier, G. A new statistical similarity measure for change detection in multitemporal SAR images and its extension to multiscale change analysis. IEEE Trans. Geosci. Remote Sens. 2007, 45, 1432-1445.

Jansing, E.D.; Albert, T.A.; Chenoweth, D.L. Two-dimensional entropic segmentation. Pattern Recognit. Lett.1999, 20, 329336.

Moser, G.; Serpico, S.B. Generalized minimum-error thresholding for unsupervised change detection from SAR amplitude imagery. IEEE Trans. Geosci. Remote Sens. 2006, 44, 2972-2982.

Liu, M.; Zhang, H.; Wang, C.; Wu, F. Change detection of multilook polarimetric SAR images using heterogeneous clutter models. IEEE Trans. Geosci. Remote Sens. 2014, 52, 74837494.

Liu W, Yang J, Zhao J, et al. A Novel Method of Unsupervised Change Detection Using Multi-Temporal PolSAR Images. Remote Sensing, 2017, 9(11): 1135.

Liu W, Yang J, Zhao J, et al. An Unsupervised Change Detection Method Using Time-Series of PolSAR Images from Radarsat-2 and GaoFen-3. Sensors, 2018, 18(2): 559.

Kapur, J.N.; Sahoo, P.K.; Wong, A.K.C. A new method for graylevel picture thresholding using the entropy of the histogram. Comput. Vis. Graph. Image Process. 1985, 29, 140.

Ketchen, D.J., Jr.; Shook, C.L. The application of cluster analysis in strategic management research: An analysis and critique. Strateg. Manag. J. 1996, 17, 441-458.

Kittler, J.; Illingworth, J. Minimum error thresholding. Pattern Recognit. 1986, 19, 41-47.

Muro J, Canty M, Conradsen K, et al. Short-term change detection in wetlands using Sentinel-1 time series[J]. Remote Sensing, 2016, 8(10), 795.

Otsu, N. A threshold selection method from gray-level histograms. Automatica 1975, 11, 23-27. 
Pham, M.-T.; Mercier, G.; Michel, J. Change Detection between SAR Images Using a Pointwise Approach and Graph Theory. IEEE Trans. Geosci. Remote Sens. 2016, 54, 2020-2032.

Rasmussen, C.E. The infinite Gaussian mixture model. Adv Neural Inf. Proc. Syst. 2000, 10, 554-560.

Stehman, S.V. Selecting and interpreting measures of thematic classification accuracy. Remote Sens. Environ. 1997, 62, 77-89.

Singh, A. Review Article Digital change detection techniques using remotely-sensed data. Int. J. Remote 1989, 10, 989-1003.

Sumaiya, M.; Kumari, R.S.S. Logarithmic Mean-Based Thresholding for SAR Image Change Detection. IEEE Geosci. Remote Sens. Lett. 2016, 13, 1726-1728.

Sun J, Yu W, Deng Y. The SAR payload design and performance for the GF-3 mission. Sensors, 2017, 17(10): 2419.

Sun, W.; Shi, L.; Yang, J.; Li, P. Building Collapse Assessment in Urban Areas Using Texture Information From Postevent SAR Data. IEEE J. Sel. Top. Appl. Earth Obs. Remote Sens. 2016, 9 , 3792-3808.

Yang, W.; Yang, X.; Yan, T.; Song, H.; Xia, G. Region-Based Change Detection for Polarimetric SAR Images Using Wishart Mixture Models. IEEE Trans. Geosci. Remote Sens. 2016, 54, 6746-6756.

Yetgin, Z. Unsupervised change detection of satellite images using local gradual descent. IEEE Trans. Geosci. Remote Sens. 2012, 50, 1919-1929.

Yousif, O.; Ban, Y. Object-Based Change Detection in Urban Areas Using Multitemporal High Resolution SAR Images with Unsupervised Thresholding Algorithms; Multitemporal Remote Sensing; Springer: Berlin, Germany, 2016,45, 89-105.

Zhao, J.; Yang, J.; Lu, Z.; Li, P.; Liu, W.; Yang, L. A Novel Method of Change Detection in Bi-Temporal PolSAR Data Using a Joint-Classification Classifier Based on a Similarity Measure. Remote Sens. 2017, 9, 846.

Zhao, J.Q.; Yang, J.; Li, P.X.; Liu, M.Y.; Shi, Y.M. An Unsupervised Change Detection Based on Test Statistic and KI from Multi-temporal and Full Polarimetric SAR Images. Int. Arch. Photogramm. Remote Sens. Spatial Inf. Sci. 2016, XLIB7, 611-615.

Zhao, J., et al., An Unsupervised Method of Change Detection in Multi-Temporal PolSAR Data Using a Test Statistic and an Improved K\&I Algorithm. Applied Sciences, 2017. 7(12): p. 1297.

Zhao, L.; Yang, J.; Li, P.; Zhang, L. Characteristics Analysis and Classification of Crop Harvest Patterns by Exploiting HighFrequency MultiPolarization SAR Data. IEEE J. Sel. Top. Appl. Earth Obs. Remote Sens. 2014, 7, 3773-3783.

Zhao, L.; Yang, J.; Li, P.; Zhang, L. Seasonal inundation monitoring and vegetation pattern mapping of the Erguna floodplain by means of a RADARSAT- 2 fully polarimetric time series. Remote Sens. Environ. 2014, 152, 426-440.
Zivkovic, Z. Improved adaptive Gaussian Mixture Model for Background Subtraction. In Proceedings of the IEEE 17th International Conference on Pattern Recognition, Cambridge, UK, 26 August 2004. 